\title{
Differentially expressed genes SNRPC and PRPF38A are potential biomarkers candidates for osteosarcoma
}

\section{Sheng Chang}

First Affiliated Hospital of Jinzhou Medical University

Yang Cao ( $\square$ caoyang_jzmu2020@126.com )

First Affiliated Hospital of Jinzhou Medical University

\section{Research}

Keywords: PRPF38A, SNRPC, Osteosarcoma, GEO, DEGs

Posted Date: April 28th, 2020

DOI: https://doi.org/10.21203/rs.3.rs-24106/v1

License: (c) (1) This work is licensed under a Creative Commons Attribution 4.0 International License.

Read Full License 
1 Differentially expressed genes SNRPC and PRPF38A are

\section{potential biomarkers candidates for osteosarcoma}

\section{SHENG CHANG ${ }^{1,2}$, YANG CAO ${ }^{1}$}

${ }^{1}$ Department of Orthopedics, The First Affiliated Hospital of Jinzhou Medical University, 2 5th Renmin Rd, Jinzhou, Guta District, Liaoning Province, China, 121000

${ }^{2}$ Postgraduate Department, Soochow University, 1 Shizi Rd, Gusu District, Suzhou Jiangsu Province,

China, 215006

Correspondence to YANG CAO, PhD, Department of Orthopedics, First Affiliated

Hospital of Jinzhou Medical University, Jinzhou 121000, Liaoning Province, China

Tel/fax: +86 416419 7717; e-mail: caoyang_jzmu2020@126.com

\section{Abstract}

Background: Osteosarcoma (osteogenic sarcoma, OS) is a primary cause of morbidity and mortality and is associated with poor prognosis in the field of orthopedic. Globally, rates of OS are highest among 15 to 25-year-old adolescent. However, the mechanism of gene regulation and signaling pathway is unknown.

Material and Methods: GSE9508, including 34 OS samples and 5 non-malignant bone samples, was gained from Gene Expression Omnibus (GEO) database. The differentially expressed genes (DEGs) were picked out by GEO2R online R soft tool. Furthermore, the protein-protein interaction (PPI) network between the DEGs was molded utilizing STRING online software. Afterward, PPI network of DEGs was constructed. Gene ontology (GO) and Kyoto Encyclopedia of Genes and Genomes (KEGG) pathway enrichment analysis of DEGs were carried out on DAVID online tool and visualized via cytoscape software. Subsequently, module analysis of PPI was performed by using MCODE app. What's more, prognosis-related genes were screened by using online databases including GEPIA, UALCAN and cBioPortal databases. Results: Totally, 671 DEGs were picked out, including 501 up-regulated genes and 170 down-regulated genes. Moreover, 22 hub genes were identified to be significantly 
expressed in PPI network (16 up-regulated and 6 down-regulated). We found that spliceosome signaling pathway may provide a potential target in OS. Furthermore, on the basis of common crucial pathway, PRPF38A and SNRPC were closely associated with spliceosome.

Conclusion: This study showed that SNRPC and PRPF38A are potential biomarkers candidates for osteosarcoma.

Key words: PRPF38A, SNRPC, Osteosarcoma, GEO, DEGs

\section{Introduction}

The early detection of OS is limited. Most patients were diagnosed at a middle or advance stage. Therefore, it is very valuable to find a biomarker for evaluation of the of OS(1-4). Recently. High-throughput biochip technology has been prevalent in screening of pre-symptomatic disease(5-7). Bioinformatics have led to using for dataintensive statistical analysis in various fields of healthcare(8-12). Development of bigdata has attracted attention in recent years(13-16). It provided new insights toward novel strategies for early testing and diagnosis of cancer(17-20). However, bioinformatics study of OS has not been catalogued. We tested GSE9508(Platform GPL676) from GEO. The dataset was divided into two part :34 OS sample and 5 nonmalignant bone sample. Microarray dataset was downloaded from GEO and analyzed via GEO2R online tool. What's more, DEGs between OS samples and non-malignant bone samples were screened by GEO2R. Moreover, DAVID version $6.8(21)$ was used to analyze KEGG pathway and GO enrichment analysis with 501 up-regulated and 170 down-regulated DEGs. Multiple PPI network was calculated by using STRING online tool version 11.0(22). Furthermore, we establish simulation of PPI network with DEGs in Cytoscape software (version 3.7.2) (23)and then 23 core genes were picked out by MCODE app(24). Next, these genes were imported into (25) cBioPortal (26)databases for significant prognosis information $(\mathrm{P}<0.05)$. However, only 8 genes were valid and then re-analyzed these central genes for KEGG pathway enrichment. Last but not least, PRPF38A and SNRPC were obtained and enriched in spliceosome pathway. The aim of present research was to provide a potential biomarker in OS. 
Microarray and DEGs. Microarray data containing OS related information were download from the GEO database (http://www.ncbi.nlm.nih.gov/gds/), including 34 OS samples and 5 non-malignant bone samples. A microarray dataset, GSE9508(GPL6076). Analyzed via GEO2R online R soft tool(27). DEGs were selected according to adjust $\mathrm{P}$-value $<0.05, \log \mathrm{FC} \mid>1$. All data were preserved in excel format. On this basis, inclusion criteria for up-regulated was $\log \mathrm{FC}>1$. Likewise, $\log \mathrm{FC}>-1$ was considered as down-regulated.

GO and KEGG pathway enrichment analysis. Two common approaches for identifying molecular function and biological process as mentioned above. Functional enrichment analysis was performed DAVID Bioinformatics resources database version 6.8(28)(http://david .ncifcrf.gov/).

Protein-protein interaction. We cluster the DEGs PPI network to identify novel gene utilizing STRING online tool version 11.0(22) (https://string-db.org). Finally, the data were saved in tab-separated values (TSV format).

Central genes module analysis. Raw data files were imported into Cytoscape3.7.2, we run Molecular Complex Detection(MCODE)(24), an app of cytoscape, to calculate the significant modules in PPI network, with the threshold set as follows: Degree cutoff=2, Node Score Cut off=0.2, Max. Depth=100, K-core=0.2.

Overall survival of prognosis-related genes. Survival prediction was performed using cBioPortal(26)(http://www.cbioportal.org/). Statistical analyses for survival were performed with log rank test. A P-values less than 5\% was considered as statistically significant. In addition, novel genes were pinpointed according to the threshold values above.

\section{Result}

Identification of DEGs in osteosarcoma. There were 34 OS samples and 5 nonmalignant samples in our present research. Totally, 671 DEGs were picked up through GEO2R online R software tool, involving 501 up-regulated (namely $\log F C>1$ ) and 170 down-regulated (namely $\log \mathrm{FC}<-1)$ (Table I).

GO and KEGG pathway enrichment analysis. GO and KEGG pathway enrichment analysis was performed using DAVID database. Within the biological process (BP) 
categories, nuclear-transcribed mRNA poly(A) tail shortening, vesicle fusion, cellular response to caffeine, female meiotic division, regulation of heart rate were dominant term for up-regulated DEGs. Down-regulated were particularly featured in translation, SRP-dependent cotranslational protein targeting to membrane, regulation of viral transcription, nuclear-transcribed mRNA catabolic process, nonsense-mediated decay, negative regulation of endopeptidase activity. For molecular function (MF)level, upregulated genes included in transcription factor activity, sequence-specific DNA binding, nucleotide binding, extracellular matrix constituent, lubricant activity, poly(A) RNA binding, RNA polymerase II regulatory region sequence-specific DNA binding. However, down-regulated genes involved in structural constituent of ribosome, protein binding, histone methyltransferase activity, U1 snRNA binding, endopeptidase inhibitor activity. In the cellular component (CC)category, eukaryotic translation initiation factor 4F complex, nucleoplasm, dendrite, cytoplasmic microtubule, primary cilium were main enrichment term for up-regulated. Whereas, the down-regulated genes were involved in photoreceptor inner segment, blood microparticle, photoreceptor outer segment, ribosome, perinuclear region of cytoplasm (Table II). KEGG pathway analysis was shown that up-regulated genes mainly play a role in spliceosome $(\mathrm{P}-\mathrm{value}<0.05)$. While the significance of identified pathway, downregulated related, was ribosome $(\mathrm{P}$-value<0.05) $($ Table III).

Protein-protein interaction. The DEGs were mapped to PPI network by using STRING (Fig. I). In addition, the network was identified via MCODE plugin in cytoscape software. Additionally, 23 central genes were screened, which include 16 upregulated and 7 down-regulated (Fig. IIA and Fig. IIB).

Overall survival of prognosis-related genes. Analysis of central gene by using cBioPortal database. A total of 8 prognosis-associated genes were identified using overall survival analysis from above database (Table IVand Fig.III).

Re-analaysis of 8 central genes by KEGG pathway enrichment analysis. A subset of 8 genes, which related to prognosis, were selected for KEGG pathway enrichment analysis. The result of the cluster shows an enrichment for terms related to spliceosome, which SNRPC and PRF38A were directly involved in (Table V and Fig. IV). 


\section{Discuss}

Early diagnosis and timely treatment contribute to improving the prognosis of sarcoma(29-32). In this study, we analyzed GSE9508 dataset downloaded from GEO database, and calculated 2 potential genes (SNRPC and PRPF38A) associated with OS through a serious of bioinformatics method. It is worth nothing that 671 DEGs were screened out after a comparative analysis between OS samples and non-malignant samples via GEO2R online $\mathrm{R}$ software $(33,34)(|\log \mathrm{FC}|>1$ and adjust $\mathrm{P}<0.05)$ including 501up-regulated and170 down-regulated DEGs. After bioinformatical analysis of GO and KEGG pathway, we acquired that 1) for BP categories, up-regulated DEGs were featured in regulation of heart rate, vesicle fusion, nuclear-transcribed mRNA poly(A) tail shortening, cellular response to caffeine, female meiotic division were dominant term for up-regulated DEGs. Down-regulated were particularly enriched in regulation of viral transcription, translation, SRP-dependent cotranslational protein targeting to membrane, nuclear-transcribed mRNA catabolic process, nonsense-mediated decay, negative regulation of endopeptidase activity; 2) for MF, up-regulated genes included in transcription factor activity, sequence-specific DNA binding, nucleotide binding, extracellular matrix constituent, lubricant activity, poly(A) RNA binding, RNA polymerase II regulatory region sequence-specific DNA binding. However, downregulated genes included in structural constituent of ribosome, protein binding, histone methyltransferase activity, U1 snRNA binding, endopeptidase inhibitor activity; 3) for $\mathrm{CC}$, up-regulated DEGs were enriched in eukaryotic translation initiation factor $4 \mathrm{~F}$ complex, nucleoplasm, dendrite, cytoplasmic microtubule, primary cilium were main enrichment term for up-regulated. Whereas, the down-regulated concentrated in photoreceptor inner segment, blood microparticle, photoreceptor outer segment, ribosome, perinuclear region of cytoplasm (Table II).

KEGG enrichment analysis provided details that up-regulated genes participated in spliceosome signaling. Based on the KEGG pathway, down-regulated gene were in ribosome (Table III, $\mathrm{P}<0.05$ ). Though PPI network construction, 23 core genes were identified (involving 16 up-regulated and 6 down- regulated) via GEPIA and UALCAN and cBioPortal databases. A total of 8 genes significantly associated with prognosis 
were screened.

Additionally, re-analysis 8 novel genes by using DAVID shown that SNRPC and PRPF38A as potential biomarkers and spliceosome had a significant, which provided a new effective target to improve the prognosis of OS patients.

Small nuclear ribonucleoprotein poly peptide C(SNRPC), is also known as U1 snRNP, which is important for recognition of pre-mRNA and assembly of spliceosome(35, 36). Another study proved that SNRPC is related to splice-site(37). Some of these protein interact with SNRPC(38). Moreover, Wang et al indicated that SNRPC, which is the main component of spliceosome(39). Lindén et al reported alternation in pre-mRNA spliceosome is a promising biomarker, which plays an important role in diagnosis of Stochastic Loewner Evolution (SLE)(40, 41).

Pre-mRNA splicing factor 38A (PRPF38A), is a center of spliceosome component, which is related to protein-protein interaction(42). Previous research proved that knock down of PRPF38A resulted in widespread intron retention (43). Whereas it was then demonstrated that knock down of PRPF38A promotes apoptosis in triple negative breast cancer(44).

To date, many studies confirm that SNRPC and PRPF38A were related to numerous types of cancer progression $(36,45-48)$. Nevertheless, studies were very few and far between in OS. Thus, these data may provide some functional information from bench to bedside.

\section{Conclusions}

In summary, our study presents SNRPC and PRPF38A were identified as potential biomarkers in OS by bioinformatical analysis. We provide a new strategy for osteosarcoma treatment, however, which should be verified in the future.

\section{Abbreviations}

OS: Osteosarcoma; GEO: Gene Expression Omnibus; DEGs: differentially expressed genes; PPI: protein-protein interaction; GO: Gene ontology; KEGG: Kyoto Encyclopedia of Genes and Genomes; MCODE: Molecular Complex Detection; BP :biological process; MF: molecular function; CC: cellular component; SNRPC: Small nuclear ribonucleoprotein poly peptide C; SLE: Stochastic Loewner Evolution; 
PRPF38A: Pre-mRNA splicing factor 38A;

180

181

182

183

184

185

186

187

188

189

190

191

192

193

\section{Ethical Approval and Consent to participate}

Not applicable.

\section{Consent for publication}

Not applicable.

\section{Availability of supporting data}

We declare that all data supporting our findings are provided in the manuscript.

\section{Conflict of Interest}

No conflict of interest declared.

\section{Acknowledgement}

We wish to thank Dr. Endo-Munoz LB for his contribution to the investigation of osteosarcoma.

\section{Funding}

Award for "Liaoning Distinguished Professor".

\section{Authors' contributions}

Dr. CY conceived and designed the study. CS contributed to data analysis and wrote the first draft of the manuscript.

\section{References}

1. Y. Suehara et al., Clinical Genomic Sequencing of Pediatric and Adult Osteosarcoma Reveals Distinct Molecular Subsets with Potentially Targetable Alterations. Clinical Cancer Research 25, 6346-6356 (2019).

2. T. Guo, H. Ma, Y. Zhou, Bioinformatics analysis of microarray data to identify the candidate biomarkers of lung adenocarcinoma. PeerJ 7, (2019).

3. F. Li et al., Identification of Key Biomarkers and Potential Molecular Mechanisms in Renal Cell Carcinoma by Bioinformatics Analysis. J Comput Biol 26, 1278-1295 (2019).

4. L. Li, X. Chen, Z. Chen, Identification of Key Candidate Genes in Dairy Cow in Response to Escherichia coli Mastitis by Bioinformatical Analysis. Front Genet 10, 1251 (2019).

5. R. Giorgi Silveira et al., MicroRNAs expressed in neuronal differentiation and their associated pathways: Systematic review and bioinformatics analysis. Brain Res Bull 157, 140-148 (2020).

6. L. Deng et al., Identification and characterization of biomarkers and their functions for docetaxel-resistant prostate cancer cells. Oncol Lett 18, 3236-3248 (2019).

7. G. Li, L. Cai, L. Zhou, Microarray gene expression profiling and bioinformatics analysis reveal key differentially expressed genes in clival and sacral chordoma cell lines. Neurological Research 41, 554-561 (2019).

8. H. Lin et al., Identification of key candidate genes and pathways in hepatitis B virus-associated 
acute liver failure by bioinformatical analysis. Medicine (Baltimore) 97, e9687 (2018).

9. L. Liu et al., Identification of Key Candidate Genes and Pathways in Endometrial Cancer by Integrated Bioinformatical Analysis. Asian Pac J Cancer Prev 19, 969-975 (2018).

10. X. X. Liu, L. Cai, F. J. Liu, An in silico analysis of human sperm genes associated with asthenozoospermia and its implication in male infertility. Medicine 97, (2018).

11. Z. Shang, Y. Zhao, K. Zhou, Y. Xu, W. Huang, PAX5 alteration-associated gene-expression signatures in B-cell acute lymphoblastic leukemia. Int J Hematol 97, 599-603 (2013).

12. S. Shen et al., Identification of core genes and outcomes in hepatocellular carcinoma by bioinformatics analysis. J Cell Biochem 120, 10069-10081 (2019).

13. C. Su et al., Microarraybased analysis of COL11A1 and TWIST1 as important differentiallyexpressed pathogenic genes between left and rightsided colon cancer. Mol Med Rep 20, 4202-4214 (2019).

14. Y. Tu et al., Identification of candidate aberrantly methylated and differentially expressed genes in thyroid cancer. J Cell Biochem 119, 8797-8806 (2018).

15. R. Wang, J. Wei, Z. Li, Y. Tian, C. Du, Bioinformatical analysis of gene expression signatures of different glioma subtypes. Oncol Lett 15, 2807-2814 (2018).

16. G. Xu et al., Gene expression profile and bioinformatics analysis revealed key molecular characteristics of chordoma-before and after TNF- a treatment. Medicine (Baltimore) 99, e18790 (2020).

17. T. Xing, T. Yan, Q. Zhou, Identification of key candidate genes and pathways in hepatocellular carcinoma by integrated bioinformatical analysis. Exp Ther Med 15, 4932-4942 (2018).

18. X. Zhang et al., Bioinformatics analysis and identification of potential genes related to pathogenesis of cervical intraepithelial neoplasia. J Cancer 11, 2150-2157 (2020).

19. Z. Hu et al., Ferritin: A potential serum marker for lymph node metastasis in head and neck squamous cell carcinoma. Oncol Lett 17, 314-322 (2019).

20. K. Jiang, H. Liu, D. Xie, Q. Xiao, Differentially expressed genes ASPN, COL1A1, FN1, VCAN and MUC5AC are potential prognostic biomarkers for gastric cancer. Oncol Lett 17, 3191-3202 (2019).

21. W. Huang da, B. T. Sherman, R. A. Lempicki, Systematic and integrative analysis of large gene lists using DAVID bioinformatics resources. Nat Protoc 4, 44-57 (2009).

22. D. Szklarczyk et al., STRING v11: protein-protein association networks with increased coverage, supporting functional discovery in genome-wide experimental datasets. Nucleic Acids Res 47, D607-D613 (2019).

23. A. Treister, A. R. Pico, Identifier Mapping in Cytoscape. F1000Res 7, 725 (2018).

24. B. Demchak et al., The Cytoscape Automation app article collection. F1000Res 7, 800 (2018).

25. D. S. Chandrashekar et al., UALCAN: A Portal for Facilitating Tumor Subgroup Gene Expression and Survival Analyses. Neoplasia 19, 649-658 (2017).

26. E. Cerami et al., The cBio Cancer Genomics Portal: An Open Platform for Exploring Multidimensional Cancer Genomics Data: Figure 1. Cancer Discovery 2, 401-404 (2012).

27. S. Yao, T. Liu, Analysis of differential gene expression caused by cervical intraepithelial neoplasia based on GEO database. Oncology Letters, (2018).

28. W. Huang da, B. T. Sherman, R. A. Lempicki, Bioinformatics enrichment tools: paths toward the comprehensive functional analysis of large gene lists. Nucleic Acids Res 37, 1-13 (2009).

29. X. Wang, M. Gu, Y. Ju, J. Zhou, PIK3C3 Acts as a Tumor Suppressor in Esophageal Squamous Cell 
Carcinoma and Was Regulated by MiR-340-5p. Med Sci Monit 26, e920642 (2020).

30. W. Hong et al., Immune-related prognosis biomarkers associated with osteosarcoma microenvironment. Cancer Cell Int 20, 83 (2020).

31. L. Endo-Munoz et al., Loss of osteoclasts contributes to development of osteosarcoma pulmonary metastases. Cancer Res 70, 7063-7072 (2010).

32. L. L. Cao et al., Novel classifiers with clinical laboratory parameters for early detection of osteosarcoma. J Clin Lab Anal, e23189 (2020).

33. C. Dong et al., Cancer stem cell associated eight gene-based signature predicts clinical outcomes of colorectal cancer. Oncol Lett 17, 442-449 (2019).

34. L. Yang et al., Bioinformatical analysis of Gene Expression Omnibus (GEO) database associates TAF7/CCNB1, TAF7/CCNA2, and GTF2E2/CDC20 pathways with glioblastoma development and prognosis. World Neurosurg, (2020).

35. L. L. Knoop, S. J. Baker, The Splicing Factor U1C Represses EWS/FLI-mediated Transactivation. Journal of Biological Chemistry 275, 24865-24871 (2000).

36. N. Ohkura, H. Yaguchi, T. Tsukada, K. Yamaguchi, TheEWS/NOR1Fusion Gene Product Gains a Novel Activity Affecting Pre-mRNA Splicing. Journal of Biological Chemistry 277, 535-543 (2002).

37. Y. Muto et al., The structure and biochemical properties of the human spliceosomal protein U1C. J Mol Biol 341, 185-198 (2004).

38. N. Ayoub, M. Alkhatatbeh, M. Jibreel, M. Ababneh, Analysis of circulating adipokines in patients newly diagnosed with solid cancer: Associations with measures of adiposity and tumor characteristics. Oncol Lett 13, 1974-1982 (2017).

39. H.-Y. Wang, L. Zhou, J.-F. Gui, Identification of a putative oocyte-specific small nuclear ribonucleoprotein polypeptide $\mathrm{C}$ in gibel carp. Comparative Biochemistry and Physiology Part B: Biochemistry and Molecular Biology 146, 47-52 (2007).

40. M. Lindén et al., Sex influences eQTL effects of SLE and Sjögren's syndrome-associated genetic polymorphisms. Biology of Sex Differences 8, (2017).

41. D. L. Armstrong et al., GWAS identifies novel SLE susceptibility genes and explains the association of the HLA region. Genes Immun 15, 347-354 (2014).

42. S. Blanton, A. Srinivasan, B. C. Rymond, PRP38 encodes a yeast protein required for pre-mRNA splicing and maintenance of stable U6 small nuclear RNA levels. Mol Cell Biol 12, 3939-3947 (1992).

43. A. E. Armstrong, D. O. Walterhouse, P. J. Leavey, J. Reichek, A. L. Walz, Prolonged response to sorafenib in a patient with refractory metastatic osteosarcoma and a somatic PDGFRA D846V mutation. Pediatr Blood Cancer 66, e27493 (2019).

44. S. Chan et al., Basal-A Triple-Negative Breast Cancer Cells Selectively Rely on RNA Splicing for Survival. Mol Cancer Ther 16, 2849-2861 (2017).

45. H. Z. Ling et al., Discovery of new serum biomarker panels for systemic lupus erythematosus diagnosis. Rheumatology (Oxford), (2020).

46. M. Koguchi et al., BMP4 induces asymmetric cell division in human glioma stem-like cells. Oncology Letters, (2019).

47. Q. Jiang, W. Feng, C. Xiong, Y. Lv, Integrated bioinformatics analysis of the association between apolipoprotein E expression and patient prognosis in papillary thyroid carcinoma. Oncol Lett 19, 2295-2305 (2020).

48. G. M. Calaf, J. Abarca-Quinones, Ras protein expression as a marker for breast cancer. Oncol 
Lett 11, 3637-3642 (2016).

Figure I. Protein-protein interaction network showing experimentally verified and predicted interaction information among the proteins encoded by the differentially expressed genes.

Figure II . A sub-network of key proteins was constructed by using Cytocape. Yellow and blue depict the up- or down-regulated differentially expressed genes, consecutively.

Figure III Prognostic survival analysis of CDC34, HNRNPA3, SRRT, HNRNPD, LRRC41, PRPF38A, SNRPC and KLHL22 genes via cBioPortal database. CDC34, cell division cycle 34; HNRNPA3, heterogeneous nuclear ribonucleoprotein A3; SRRT, serrate RNA effector molecule; HNRNPD, heterogeneous nuclear ribonucleoprotein D; LRRC41, leucine rich repeat containing 41; PRPF38A, pre-mRNA processing factor 38A; SNRPC, small nuclear ribonucleoprotein polypeptide C; KLHL22, kelch like family member 22.

Figure IV Significantly enriched pathway terms of DEGs in osteosarcoma. KEGG pathway annotations of the spliceosome pathway. Red star-marked means significant genes.

Table I All 671 DEGs were detected from GSE9508, involving 501 up-regulated and 170 down regulated genes in OS samples compared to non-malignant sample.

Table II GO enrichment analysis of DEGs in osteosarcoma.

Table III KEGG enrichment analysis of DEGs in osteosarcoma.

Table IV The prognosis-related information of 23 central genes.

Table V KEGG pathway enrichment analysis with 2 novel genes. 
Figures

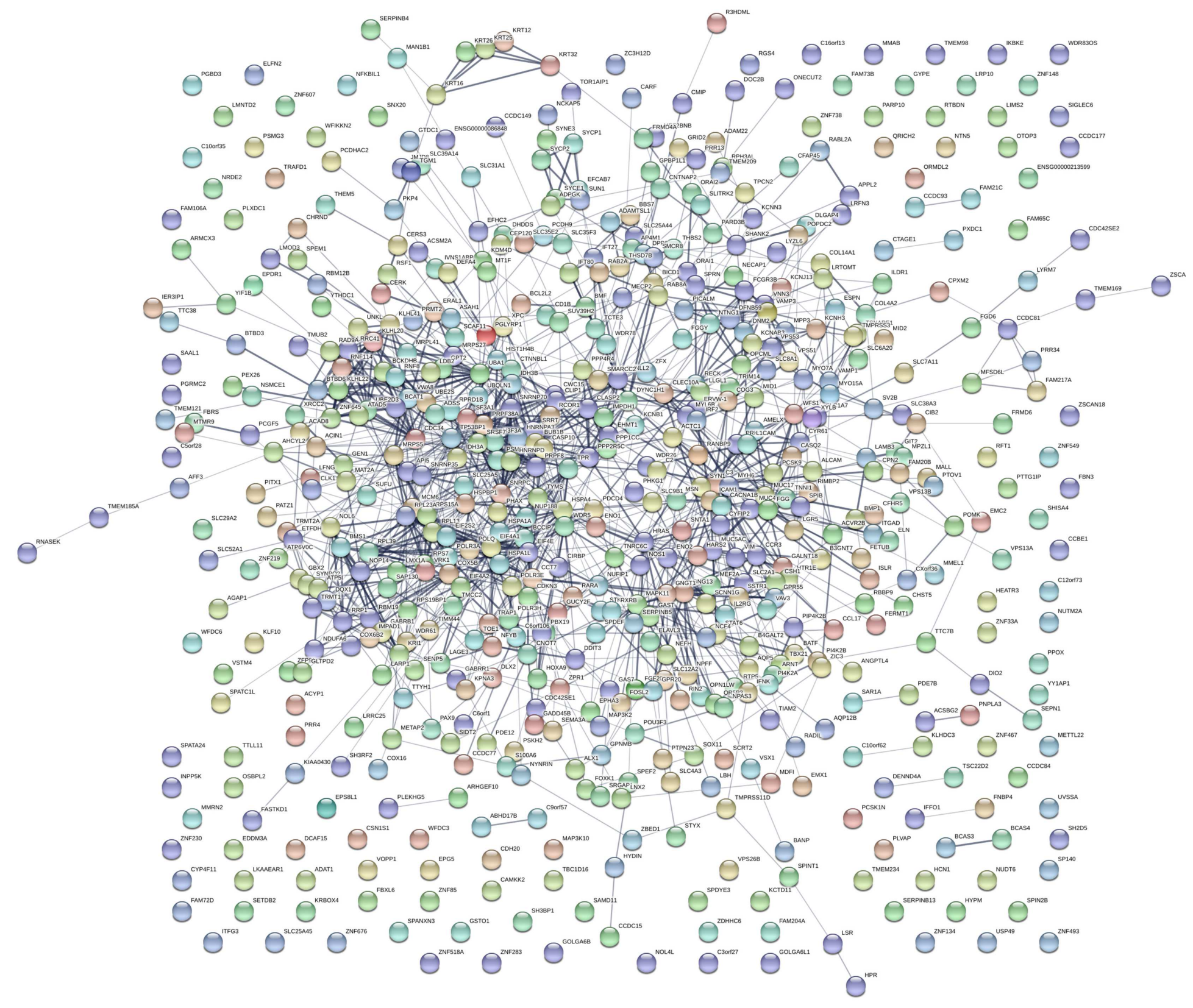

Figure 1

Protein-protein interaction network showing experimentally verified and predicted interaction information among the proteins encoded by the differentially expressed genes. 


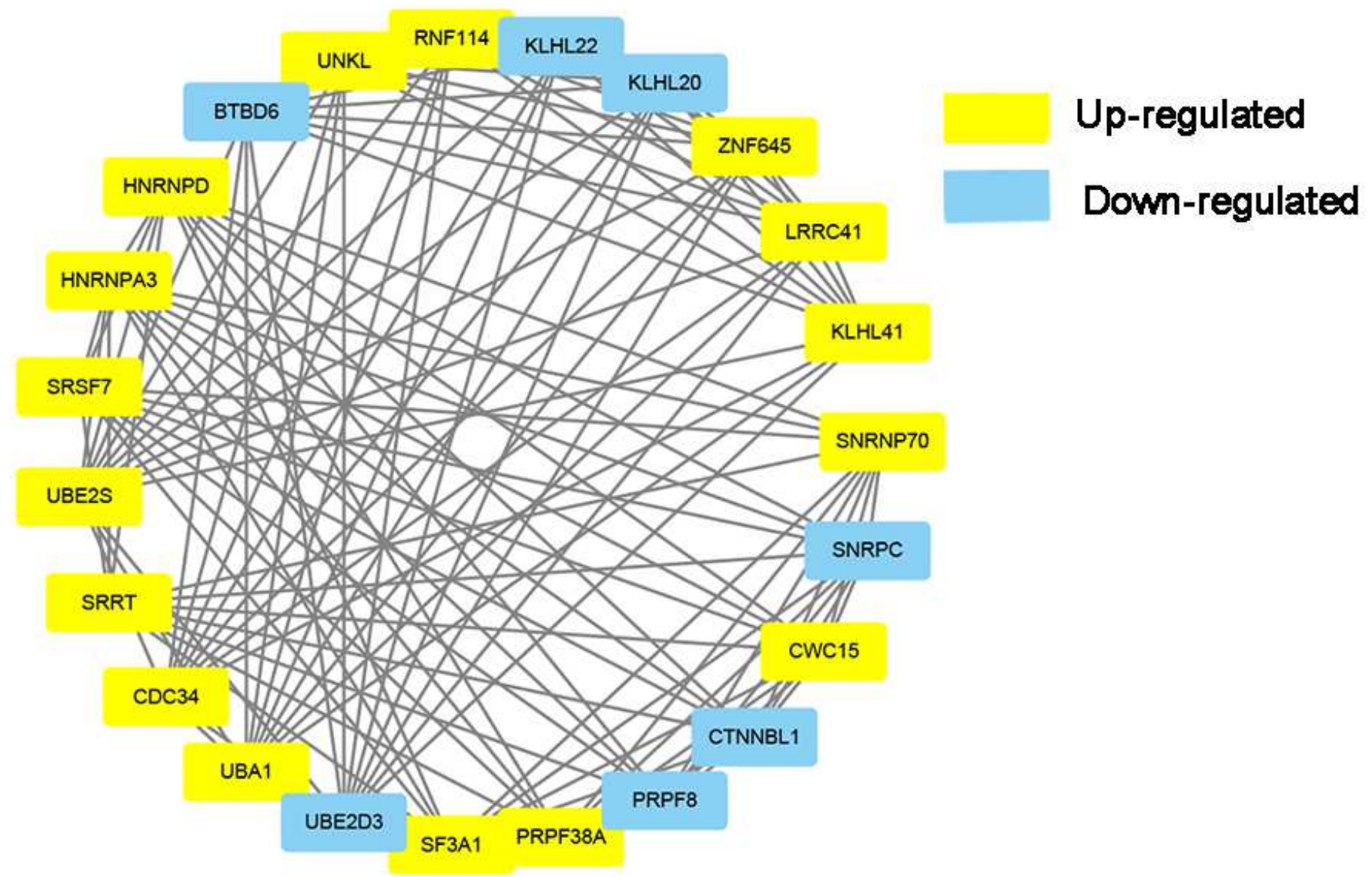

Figure 2

A sub-network of key proteins was constructed by using Cytocape. Yellow and blue depict the up- or down-regulated differentially expressed genes, consecutively. 

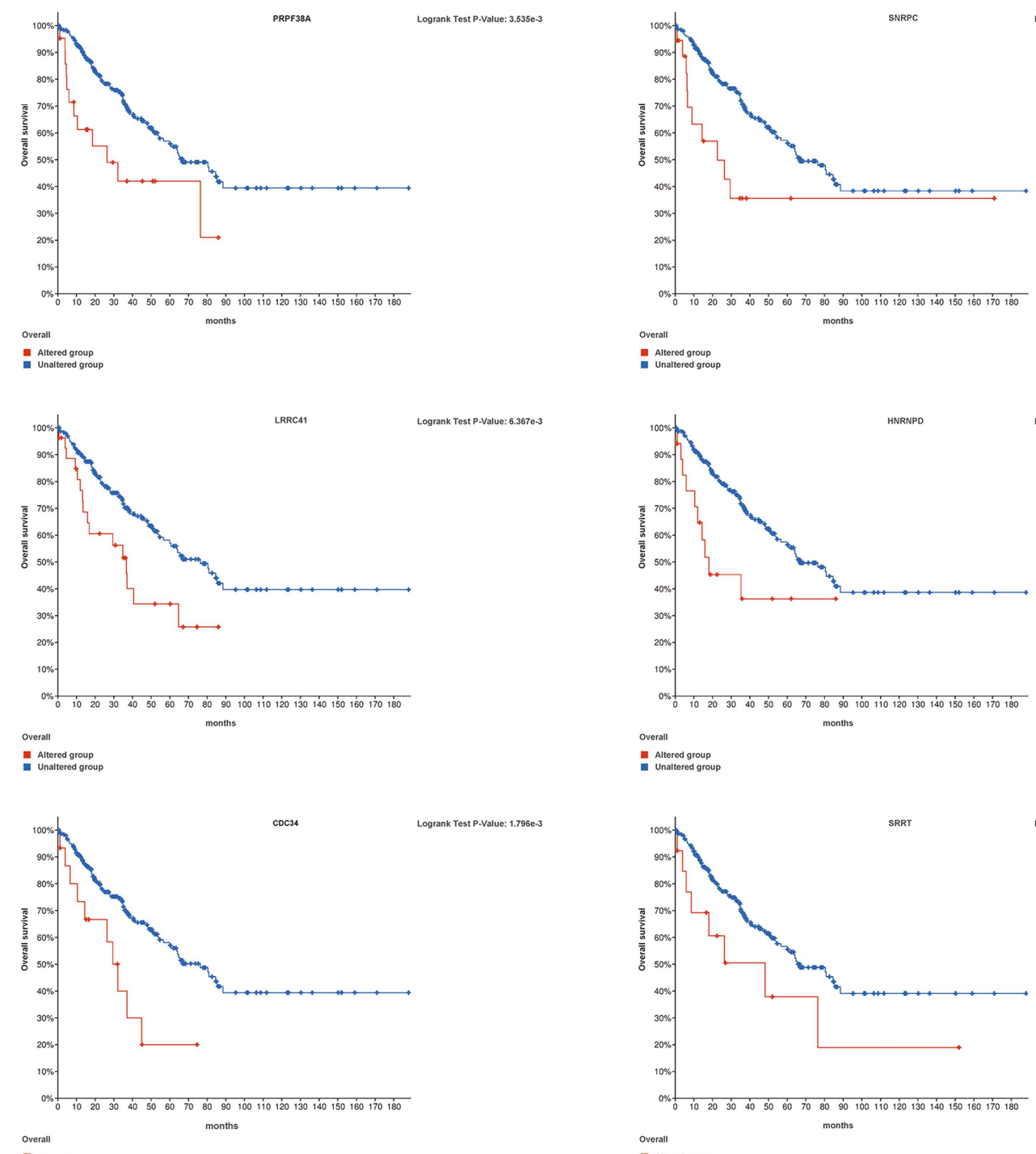

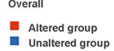
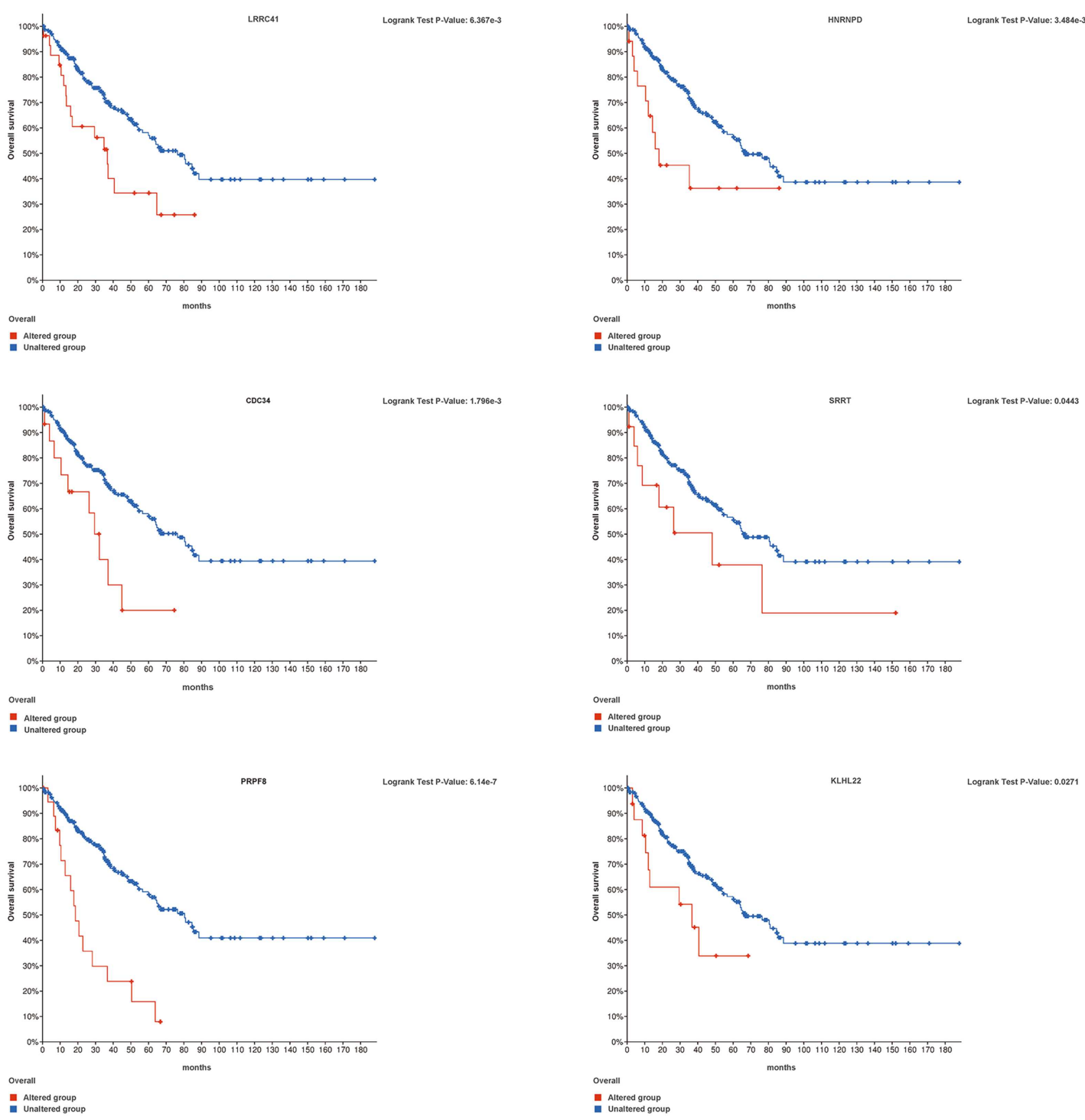

\section{Figure 3}

Prognostic survival analysis of CDC34, HNRNPA3, SRRT, HNRNPD, LRRC41, PRPF38A, SNRPC and KLHL22 genes via cBioPortal database. CDC34, cell division cycle 34; HNRNPA3, heterogeneous nuclear ribonucleoprotein A3; SRRT, serrate RNA effector molecule; HNRNPD, heterogeneous nuclear ribonucleoprotein D; LRRC41, leucine rich repeat containing 41; PRPF38A, pre-mRNA processing factor 38A; SNRPC, small nuclear ribonucleoprotein polypeptide C; KLHL22, kelch like family member 22. 
SPLICEOSOME

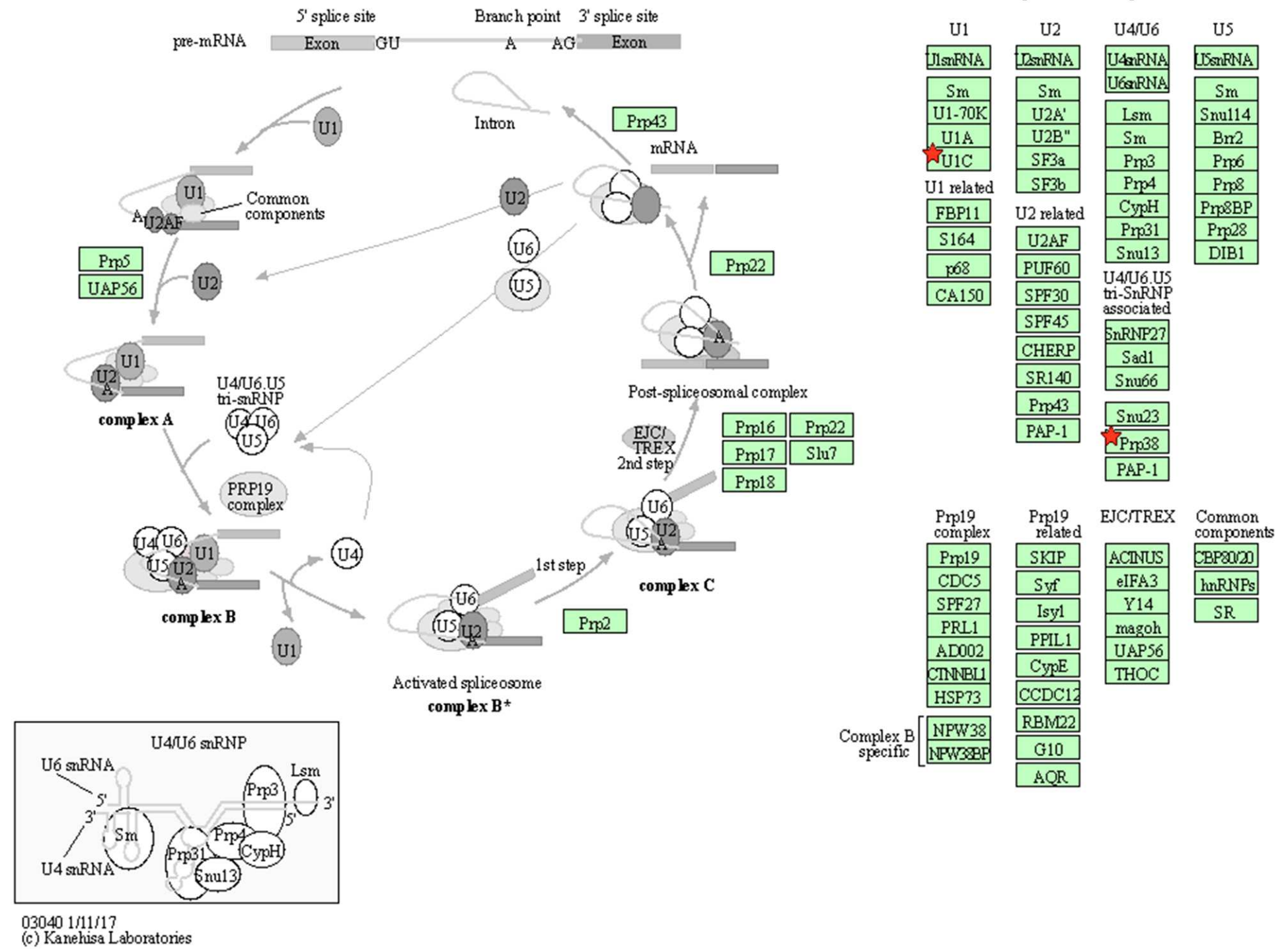

\section{Figure 4}

Significantly enriched pathway terms of DEGs in osteosarcoma. KEGG pathway annotations of the spliceosome pathway. Red star-marked means significant genes.

\section{Supplementary Files}

This is a list of supplementary files associated with this preprint. Click to download.

- Table4.pdf

- Table5.pdf

- Table1.pdf

- Table3.pdf

- Table2.pdf 